\title{
The Society for Computer Applications in Radiology
}

$\mathbf{T}$ HE TENTH CONFERENCE on Computer Applications in Radiology and the Fourth Conference on Computer-Assisted Radiology will be combined as S/CAR '90, a Symposium for Computer-Assisted Radiology. It will take place June 13-16, 1990 in Anaheim, CA. This meeting is sponsored by The Society for Computer Applications in Radiology (SCAR) and the organizing committee for ComputerAssisted Radiology (CAR). The conference represents the merger of two series of well-established, highly successful meetings. For more than 20 years the American College of Radiology (ACR) has sponsored biennial conferences on computers in radiology. In 1988 the ACR was joined by the Radiology Information System Consortium (RISC) to sponsor the ninth biennial conference in this series. Now SCAR is proud to sponsor the 10th meeting along with the ACR and join forces with the Fourth Conference on Computer-Assisted Radiology, previously sponsored by CAR in Europe. The leaders of the major computer/radiology groups are coordinating our meetings and activities so that there are no scheduling conflicts and so that there is maximum attendance and benefit for our meeting attendees and participants.

S/CAR ' 90 will have a wide assortment of activities including tutorials and workshops, special poster sessions with authors present for questions and discussion, scientific presentations, and many technical exhibits featuring the latest equipment and services. For more detailed information concerning S/CAR '90 contact Janice Ford, Manager, Continuing Education, Hospital of the University of Pennsylvania, 3400 Spruce St, Philadelphia, PA 19104; Phone: 215-6626904.

The Governance Committee of SCAR has approved a new category of Junior Membership, with reduced annual dues of $\$ 50$ for individuals in training programs. We want to involve all levels of interested persons in SCAR's activities. Please let your residents, graduate students, medical students, and so forth know about this new membership category. Also, please be sure to fill out the SCAR questionnaire sent to all members. It has been designed to be as easy to fill out as possible, while giving us as much useful information as possible about our membership. I am particularly interested in your opinions about how SCAR should be structured to provide you with the most personal benefit, and what activities SCAR should be undertaking in the educational and scientific arena.

Tim B. Hunter, $M D$

Chairman, SCAR

Department of Radiology Arizona Health Sciences Center

Tucson, AZ 85724 